\title{
Bauer, Dieter R., Becher, Matthias, Plassmann, Alheydis, Welf IV. - Schlüsselfigur einer Wendezeit. Regionale und europäische Perspektiven
}

\section{Philippe Depreux}

\section{OpenEdition}

\section{Journals}

Édition électronique

URL : http://journals.openedition.org/ifha/2106

DOI : $10.4000 /$ ifha. 2106

ISSN : 2198-8943

Éditeur

IFRA - Institut franco-allemand (sciences historiques et sociales)

Référence électronique

Philippe Depreux, «Bauer, Dieter R., Becher, Matthias, Plassmann, Alheydis, Welf IV. - Schlüsselfigur einer Wendezeit. Regionale und europäische Perspektiven », Revue de I'IFHA [En ligne], Date de recension, mis en ligne le 01 janvier 2010, consulté le 22 septembre 2020. URL : http://journals.openedition.org/ ifha/2106 ; DOI : https://doi.org/10.4000/ifha.2106

Ce document a été généré automatiquement le 22 septembre 2020.

(CIFHA 


\title{
Bauer, Dieter R., Becher, Matthias, Plassmann, Alheydis, Welf IV. - Schlüsselfigur einer Wendezeit. Regionale und europäische Perspektiven
}

\author{
Philippe Depreux
}

C'est bien, comme le souligne le titre, à une figure clef de l'histoire de l'Empire vers la fin du XIe siècle, dont le rôle se déclina à la fois à l'échelle régionale et sur l'échiquier politique européen, qu'est consacré le volume édité par D. BAUER et M. BECHER. L'ouvrage comporte des communications présentées lors d'un colloque tenu à Weingarten, l'un des hauts lieux de la memoria des Welfs (cf. Sönke LORENZ, " Weingarten und die Welfen », p. 30-55), dont le nom est à l'origine de celui du parti guelfe rassemblant les partisans de la papauté. Cette rencontre fut tenue en 2001, à l'occasion du 900e anniversaire supposé de la mort du duc de Bavière, même si en réalité, les actes du colloque montrent que c'est d'une année plus tard - donc en 1102 qu'il faut dater son décès, survenu alors qu'il s'était croisé (Marie-Louise FAVREAULILIE : «Welf IV. und der Kreuzeug von 1101 », p. 420-447).

Les grandes lignes de la place qu'occupe Welf IV dans l'histoire de l'Empire sont esquissées en introduction par Bernd SCHNEIDMÜLLER, dont le texte reprend la conférence inaugurale qu'il prononça à Ravensburg à l'attention du grand public (« Welf IV. 1101-2001. Kreationen fürstlicher Zukunft », p. 1-29). Bien qu'aucun article ne soit explicitement consacré aux rapports entre le duc de Bavière, qui fut l'un des meneurs de l'opposition princière à Henri IV, et les papes (notamment Urbain II, qui avait eu l'occasion de connaître personnellement Welf IV lors d'une mission en tant que légat du pape Grégoire VII et qui, le lendemain même de son élection au souverain pontificat, en informa le duc parmi les premiers), cette question sous-tend l'ensemble du volume, placé sous le signe de la réforme grégorienne en Souabe et en Bavière, qu'il s'agisse du soutien apporté par le duc à cet aggiornamento des institutions ecclésiales étudié par feu Johannes LAUDAGE ("Welf IV. und die Kirchenreform des 11. 
Jahrhunderts ", p. 280-313), de sa coopération avec l'évêque de Constance, Gebhard III, qu'il contribua à faire élire en 1084 - ainsi que le montre Helmut MAURER, qui en analyse l'action pastorale (« Bischof Gebhard III. von Konstanz und Welf IV. als Häupter der süddeutschen Reformpartei », p. 314-338) - ou de la fondation d'un établissement conventuel sous le signe de la réforme, tels les chanoines réguliers de Rottenbuch dont les origines sont étudiées par Franz FUCHS (« Die Anfänge Rottenbuchs », p. 261-279). Il en va là moins de choix dictés par des convictions profondes, toujours délicates à analyser, que du résultat d'un certain opportunisme politique ; cela semble assez évident - et fut souligné par certains observateurs médiévaux. La revendication de l'héritage de Welf III (éludé dans la Genealogia Welforum) par son neveu alors que la communauté - à cette époque féminine - d'Altdorf avait été instituée par le défunt comme son légataire universel plaça le jeune aristocrate "italien » (en tant que fils d'Albert Azzo II d'Este, il était promis à un avenir essentiellement milanais - cf. Katrin BAAKEN : «Welf IV., der 'geborene Italiener' als Erbe des Welfenhauses », p. 199-225) au cœur des jeux de pouvoir des princes de l'Allemagne du sud, dont la coalition est étudiée par Thomas ZOTZ : "Der südwestdeutsche Adel und seine Opposition gegen Heinrich IV. » (p. 339-359). Welf IV n'en perdit pas pour autant le sens des rapports de force en Italie, comme en témoigne le mariage - indéniablement arrangé - de son fils, Welf $\mathrm{V}$, avec Mathilde de Toscane, qui aurait pu être sa mère : au début du pontificat d'Urbain II, le service de la politique de ce dernier, dont le duc de Bavière deviendrait d'ailleurs le vassal en 1093 (cf. l'article de H. Maurer, p. 331-332), primait sur toute considération dynastique. Cette union est analysée par Elke GOEZ : "Welf V. und Mathilde von Canossa » (p. 360-381).

Ce volume permet non seulement de bien mettre en lumière divers aspects de l'action régionale des Welfs (Heinz DOPSCH : «Welf III. und Kärnten, p. 84-128 ; Karel HRUZA : « Omne patrimonium suum cum ministerialibus - Zur Herkunft welfischer Dienstmannen in Oberschwaben am Beispiel der Herren von Wallsee ", p. 382-419), mais aussi la manière dont Welf s'émancipa du contrôle royal, question étudiée par Hubertus SEIBERT ( Vom königlichen dux zum Herzog von Bayern. Welf IV. und der Südosten des Reiches ", p. 226-260). Reste une question fondamentale au regard de la place qu'occupent les Welfs dans l'histoire de la conscience familiale aristocratique, dont les travaux pionniers de Karl Schmid et Otto Gerhard Oexle ont montré l'importance. Plusieurs contributions y ont, plus ou moins directement, trait. En matière de généalogie, il y a débat sur la continuité entre le père de l'impératrice Judith, la famille de Henri « mit dem goldenen Wagen » et la famille d'Este - il s'agit ici non pas la continuité biologique, mais de la conscience d'appartenir à une même famille. À cet égard, on peut considérer avec Werner HECHBERGER que, bien que la veuve de Welf II se soit opposée à la disparition - sous le régime de la mainmorte - de l'héritage familial en imposant son petit-fils, Welf IV, comme héritier (de manière significative, c'est une femme qui « sauve » l'identité familiale !), la conscience d'une telle identité familiale ou de son évidence - est essentiellement le produit d'une construction a posteriori (" Die Erbfolge von 1055 und das welfische Selbstverständnis ", p. 129-155). En la matière, le nom est d'importance. Or Matthias BECHER montre non seulement la part de hasard qu'il y a dans la succession d'homonymes au cours du XIe siècle, mais aussi et surtout, il montre que la signification du nom de Welf est plus ambiguë que l'historiographie traditionnelle en aval d'Henri le Lion veut bien le faire croire. Il est séduisant de penser qu'il s'agit originellement d'un surnom (le « chiot » ou " louveteau ») peut-être porté par le comte Eticho au IXe siècle ; ce n'est que bien plus tard que se greffa là-dessus 
l'étymologie plus romaine établissant un lien avec Catilina et le lion (« Der Name 'Welf' zwischen Akeptanz und Apologie. Überlieferung zur frühen welfischen Hausüberlieferung ", p. 156-198).

Nombre de contributions dans cet ouvrage se réfèrent à l'Historia Welforum, un monument de la mémoire aristocratique, justement célèbre - signalons pour compléter cette présentation que cette source a été récemment éditée à nouveaux frais et traduite par Matthias BECHER : Quellen zur Geschichte der Welfen und die Chronik Burchards von Ursberg, Darmstadt 2007. La question de l'originalité de ce texte est au cœur de l'exposé d'Alheydis PLASSMANN : «Die Welfen-Origo, ein Einzelfall ?»(p. 56-83). L'auteur compare l'Historia Welforum avec l'histoire des comtes d'Anjou (les Gesta consulum Andegavorum) et celle des comte de Flandre (la Flandia Generosa), pour mettre en évidence certaines similitudes typologiques, mais d'une façon assez artificielle qui néglige malheureusement tout l'intérêt que revêtirait une analyse sollicitant à part égale l'apport d'une analyse littéraire sur un thème où la production épique, par exemple, offrirait matière à de fructueuses comparaisons ; quant à l'origine potentiellement troyenne des Welfs (p. 61-62), elle ne peut être étudiée de manière déconnectée de celle dont les Francs se prévalaient et de son exploitation ultérieure (qu'on se reporte pour cela aux travaux de Colette Beaune).

Dans les actes de ce colloque, il est à mainte reprise fait allusion à certains manuscrits : quelques planches d'illustrations auraient été les bienvenues, ainsi que des cartes réalisées spécialement pour l'occasion et plus précises ou adaptées au texte que ne le sont les deux cartes reproduites aux p. 448 et 449. Par ailleurs, toutes les dates mentionnées à la p. 95 et au premier paragraphe de la p. 96 doivent être rajeunies d'un siècle. Nonobstant ces remarques, il s'agit d'un volume de haute tenue qui, tout à la fois, propose une synthèse solide sur la situation politique en Allemagne du sud dans la seconde moitié du XIe siècle et offre de nouvelles perspectives de recherche sur la base d'analyse particulièrement fouillées.

Philippe Depreux (Université de Limoges / Institut universitaire de France) 